\title{
Influence of the Adsorption Energy on the Dielectric Contribution to the Anchoring Energy of Nematic Liquid Crystals
}

\author{
H.A. Pereira ${ }^{1}$, L.R. Evangelista ${ }^{1,2}$, D. Olivero $^{2}$, and G. Barbero ${ }^{2}$ \\ ${ }^{1}$ Departamento de Física - Universidade Estadual de Maringá, \\ Av. Colombo 5790, 87020-900 Maringá, PR, Brazil \\ 2 Dipartimento di Fisica del Politecnico and \\ I. N. F. M., Corso Duca degli Abruzzi, 24-10129 Torino, Italy
}

Received on 30 November, 2001

\begin{abstract}
The influence of the ionic adsorption on the anchoring energy of a nematic liquid crystal sample is investigated. We determine the behavior of the anchoring energy as a function of the thickness of the sample, and as a function of the adsorption energy of ions. We show that the contribution to the anchoring energy, due to ionic adsorption, can be of the same order of magnitude of the bare anchoring strength. Our analysis generalizes similar calculations previously published by incorporating the effect of adsorbed charges on the potential and field profiles in the sample.
\end{abstract}

\section{Introduction}

From the practical point of view it is important to know the alignment of a nematic liquid crystal sample when it is in contact with a solid substrate. The uniform alignment of liquid crystals in this case is crucial for display applications and other liquid crystalline devices [1]. Therefore, the liquid crystal surface properties and, in particular, the characteristics features of the anchoring of the liquid crystals are very important for the performance of the liquid crystal devices since the strength of the anchoring affects the threshold characteristics of the sample [2]. It is known that in many real samples the anchoring energy can be thickness dependent $[3,4]$ and can be also dependent on the bias voltage [5]. However, the precise nature and the origin of the anchoring energy in nematic liquid crystals is still a subject of many fundamental and experimental studies and cannot be considered as a solved problem $[3,6]$. To explain the thickness dependence of the anchoring energy found in some real nematic liquid crystal samples the phenomenon of the selective ion adsorption has been invoked [7-10]. The influence of the selective ion adsorption on the anisotropic part of the anchoring energy strength has been discussed by several authors in the last years $[4,5,11-16]$. According to this point of view $[8,9]$, the adsorption phenomenon is responsible for an ionic separation inside the liquid. To this charge separation is connected an electric field distribution across the sample. The coupling of this field with the dielectric and flexoelectric properties of the liquid crystal gives rise to a dielectric energy density, localized near to the limiting surfaces, on mesoscopic thicknesses. This energy can be considered as a surface energy, which renormalizes the anisotropic part of the interfacial energy characterizing the interface nematic liquid crystal - substrate. The distribution of the field across the sample and its connection with the adsorption energy has been discussed in $[10,17,18]$. In the case in which the phenomenon of selective adsorption is absent, the effect discussed above is also absent. Summarizing: the charge separation induced by an external field gives rise to a spatial dependent electric field inside the sample. This electric field couples with the dielectric and flexoelectric properties of the nematic media.

Recently, a complete model for the adsorption phenomena in an isotropic liquid was proposed, in which the effect of external fields was taken into account in a successful way [18]. In this model, the presence of positive and negative charges was taken into account, but the adsorption was considered as selective with respect to the positive ones, i.e., the adsorption energy for the negative charges was taken as infinite. It was shown also that, according to the value of the external difference of potential applied to the sample by an external power supply, two regimes are possible. The border 
between the two regimes is fixed by the surface density of ions, originated from the chemical dissociation of the impurities present in the liquid. In the low voltage region the electric field in the sample changes sign. On the contrary, in the high voltage region, the electric field is everywhere oriented in the same direction.

Very recently, the dieletric contribution to the anchoring strength in a nematic liquid crystal sample was analyzed [19]. The analysis was performed in the hypothesis that the electrodes are perfectly blocking and that there is no selective ion adsorption. The proposed theory predicts an effective anchoring energy dependent on the applied voltage, in good agreement with experimental results. According to the sign of the dielectric anisotropy and of the flexoelectric coefficient the dependence of the anchoring energy strength with the applied voltage can be monotonic or not. For large applied voltage the effective anchoring energy strength tends to a constant value.

In this paper, we focus our attention on the effect of an adsorption energy on the anchoring energy of dielectric origin. More precisely, we explicitly consider the phenomenon of selective ionic adsorption and its influence on the anchoring energy of an NLC sample. First, we recall the formalism proposed in Ref. [19] to analytically determine the dielectric contribution to the anchoring energy. After that, we present the general equations governing the field distribution in the sample, when the phenomenon of ionic adsorption is taken into account, as is done in Ref. [18]. Finally, we apply this formalism to determine the behavior of the anchoring energy as a function of the adsorption energy and as a function of the thickness of the sample. We show that the trend and the order of magnitude of the anchoring energy of dielectric origin, theoretically predicted in our analysis, as a function of the thickness of the sample, is in good agreement with experimental results. We show furthermore that the magnitude of the anchoring energy of dieletric origin is strongly affected by the adsorption energy of positive ions and presents a nonmonotonic behavior as a function of this energy. It is also shown that the flexoelectric contribution plays a dominant role in establishing the correct order of magnitude for the anchoring energy.

\section{Dielectric contribution to the anchoring strength}

Let us consider a nematic liquid crystal limited by two solid surfaces, at a distance $d$ apart. The $z$-axis is normal to the bounding surfaces, with the origin in the middle of the sample. The liquid is supposed to contain ions and submitted to an external field. As we shall show below, in this case the electric field profile inside the sample is $z$-dependent and will be denoted by $E(z)$. The field $E(z)$ differs from the one in the bulk, $E_{B}=E(0)$, mainly close to the bounding surfaces, due to the presence of the ions, as it will be discussed in details later. If the liquid is an anisotropic fluid, as a nematic liquid crystals, the presence of the ionic charges gives rise to a surplus of surface energy characterizing the nematic liquid crystal-substrate interface. To evaluate the dielectric contributions to the surface energy we have to take into account the coupling of the external field with the dielectric anisotropy, $f_{D}(E)$, and with the flexoelectric properties of the liquid crystal, $f_{Q}(E)[8,9]$.

The quantities $f_{D}(E)$ and $f_{Q}(E)$, which are bulk energy densities, are given by

$$
f_{D}(E)=-\frac{1}{2} \epsilon_{a} E^{2}(z) \cos ^{2} \theta
$$

and

$$
f_{Q}(E)=e\left(\cos ^{2} \theta-\frac{1}{3}\right) \frac{d E(z)}{d z},
$$

where $\theta=\cos ^{-1}(\vec{n} \cdot \vec{z})$ is the angle formed by the director field $\vec{n}$ with the $z$-axis. Furthermore $\epsilon_{a}=\epsilon_{\|}-\epsilon_{\perp}$ is the dielectric anisotropy ( $\|$ and $\perp$ refer to $\vec{n}$ ), and $e=e_{11}+e_{33}$ the total flexoelectric coefficient.

Let us indicate by $E_{B}=E(0)$ and by $E_{S}=E(d / 2)$ the values of the electric field in the middle and at the surface of the sample, respectively. The dielectric energy, per unit surface, is

$$
F_{E}=\int_{-d / 2}^{d / 2}\left[f_{D}(E)+f_{Q}(E)\right] d z .
$$

This quantity can be written as

$$
\begin{aligned}
F_{E} & =\int_{-d / 2}^{d / 2}\left[f_{D}(E)-f_{D}\left(E_{B}\right)+f_{Q}(E)-f_{Q}\left(E_{B}\right)\right] d z \\
& +\int_{-d / 2}^{d / 2}\left[f_{D}\left(E_{B}\right)+f_{Q}\left(E_{B}\right)\right] d z .
\end{aligned}
$$


Taking into account that $E(z)-E_{B}$ is different from zero, practically, only in two surfaces layers of mesoscopic thickness, for the presence of the ions, we can put Eq. (4) in the form

$$
F_{E}=f_{1}+f_{2}+\int_{-d / 2}^{d / 2}\left[f_{D}\left(E_{B}\right)+f_{Q}\left(E_{B}\right)\right] d z
$$

where

$$
f_{1}=-\frac{1}{2} \epsilon_{a} \cos ^{2} \theta_{1} \int_{-d / 2}^{0}\left[E^{2}(z)-E_{B}^{2}\right] d z-e\left(\cos ^{2} \theta_{1}-\frac{1}{3}\right)\left(E_{S}-E_{B}\right),
$$

and

$$
f_{2}=-\frac{1}{2} \epsilon_{a} \cos ^{2} \theta_{2} \int_{0}^{d / 2}\left[E^{2}(z)-E_{B}^{2}\right] d z+e\left(\cos ^{2} \theta_{2}-\frac{1}{3}\right)\left(E_{S}-E_{B}\right),
$$

with $\theta_{1}=\theta(-d / 2)$ and $\theta_{2}=\theta(d / 2)$. $f_{1}$ and $f_{2}$ are the dielectric contributions, due to the ions, to the surface energy. The relevant anchoring energy strengths, coinciding with the coefficient of $\cos ^{2} \theta_{i}(i=1,2)$, are then

$$
W_{D}=-\frac{1}{2} \epsilon_{a} \int_{0}^{d / 2}\left[E^{2}(z)-E_{B}^{2}\right] d z
$$

and

$$
W_{Q}= \pm e\left(E_{S}-E_{B}\right)
$$

where \pm refer to $z= \pm d / 2$. Once the electric field distribution across the sample is known, one can directly evaluate the contribution of dielectric origin to the anchoring energy of a nematic liquid crystal sample, by means of Eqs. (8) and (9). It is necessary to reinforce the fact that these equations represent only the contribution of dielectric origin to the anchoring energy. There is a localized surface energy which does not depend on the presence of ions in the sample. It is an intrinsic characteristic of the interface. In this sense the dielectric contribution renormalizes this "bare" anchoring energy, $W_{0}$, giving rise to an effective anchoring energy that can be written in the form

$$
W_{\text {eff }}=W_{0}+W_{D}+W_{Q} .
$$

In this paper we focus our attention only on the contribution of dielectric origin in order to emphasize the necessity to take into account the presence of ions and of an adsorption energy on the anchoring energy of an NLC sample.

In order to show the importance of the above formalism, let us calculate $W_{D}$ and $W_{Q}$ by explicitly taking into account the presence of the ions in the sample. To do this we have to establish the electric field profile inside it. The equations governing the electric field distributions were established in Ref. [18]. However, it is convenient to present them here in details, due to the extensive use that will be made along this paper.

\section{The model for the electric field distributions}

The model deals with a cell in the shape of a slab of thickness $d$, filled with a liquid characterized by a dielectric constant $\epsilon$, but containing impurities. These impurities are the source of the ions by means of a chemical reaction, whose activation energy is indicated by $E_{\text {activation }}$. The activation energy $E_{\text {activation }}$ can be identified with the electrostatics interaction energy between the positive and negative ions resulting from the dissociation of the particle. We consider the case in which the surfaces are identical, but in the hypothesis that the adsorption energy for positive ions is different from the one for negative ions in each surface. We use a Cartesian reference frame whose $z$-axis is normal to the limiting walls, located at $z= \pm d / 2$. We assume that all the physical quantities entering in the model are only $z$ dependent. The distribution of charges produced by the ionic adsorption gives rise to a liquid which is locally charged, but globally neutral. We denote by $n_{0}$ the bulk density of impurities for an infinite sample. The equilibrium distribution of the bulk density of non dissociated impurities is given by

$$
n_{b}=n_{0} e^{\mu},
$$

where $\mu$ is the chemical potential in $k_{B} T$ units. Furthermore, the bulk densities of positive and negative ions are given by

$$
n_{ \pm}(z)=n_{0} e^{\mu-\Delta \mp \psi(z)},
$$


where $\Delta=E_{\text {activation }} / k_{B} T$ is the activation energy and $\psi(z)=q V(z) / k_{B} T$ is the electrostatic energy of the charge $q$, in $k_{B} T$ units. This means that in our formalism the surface electrical potential is also measured in units of $k_{B} T / q$ and, for convenience, the quantity $\psi_{S}$ will be henceforth referred simply as the "surface potential".

The surface density of adsorbed ions of a given sign is given by

$$
\sigma_{i, \pm}=N_{ \pm} e^{\mu-A_{ \pm} \mp \psi_{i}}
$$

where $i=1,2$ refers to the surfaces ( 1 for $z=-d / 2$ and 2 for $z=d / 2$ ) and $N_{ \pm}$are the surface densities of sites where the ions $(+$ and -$)$ can be adsorbed. In the above expression we have introduced the adsorption energies $A_{ \pm}$(for + and - ions) measured in $k_{B} T$ units. The adsorption energy can be identified with the electrostatic interaction energy of an adsorbed ion with its image in the substrate (physical adsorption) [22]. Finally, in (13) $\psi_{1}=\psi(z=-d / 2)$ and $\psi_{2}=\psi(z=d / 2)$ are the values of the surface potentials. We work in the hypothesis that only the internal charges move to the surface. The external charges supplied to the system are supposed to remain in the surface, separated from the liquid by blocking electrodes. We assume, furthermore, that $N_{+}=N_{-}=N$ and, in this manner, the actual surface density of adsorbed ions is given by

$$
N_{i}=N e^{\mu}\left(e^{-A_{+}-\psi_{i}}+e^{-A_{-}+\psi_{i}}\right) .
$$

The actual surface charge density due to the adsorption phenomenon is

$$
Q_{i}=q\left(\sigma_{i,+}-\sigma_{i,-}\right)=q \sigma_{i} .
$$

Notice that the surface densities of charges will have both the internal contribution (coming from the ionic charges present in the liquid) and the external contribution (coming from the external power supply).

To establish the fundamental equations governing the equilibrium distributions of charges and fields in our model we start by imposing the conservation of the number of particles in the system. This requirement, per unit surface, is written as

$$
\frac{N_{+}+N_{-}}{2}+N_{B}+\frac{\sigma_{1}+\sigma_{2}}{2}=n_{0} d
$$

where

$$
N_{ \pm}=\int_{-d / 2}^{d / 2} n_{ \pm}(z) d z, \quad \text { and } \quad N_{B}=\int_{-d / 2}^{d / 2} n_{b}(z) d z=n_{b} d .
$$

Using the definitions of $n_{ \pm}(z)$, given by (12), and $\sigma_{i}$, given by (13), it is possible to rewrite Eq. (16) in the form

$$
e^{\mu}\left\{n_{0} e^{-\Delta} \int_{-d / 2}^{d / 2} \cosh \psi(z) d z+n_{0} d+\frac{N}{2}\left[e^{-A_{+}}\left(e^{-\psi_{1}}+e^{-\psi_{2}}\right)+e^{-A_{-}}\left(e^{\psi_{1}}+e^{\psi_{2}}\right)\right]\right\}=n_{0} d .
$$

In this case the chemical potential is given by

$$
e^{-\mu}=1+\frac{1}{2 n_{0} d}\left[e^{-A_{+}}\left(e^{-\psi_{1}}+e^{-\psi_{2}}\right)+e^{-A_{-}}\left(e^{\psi_{1}}+e^{\psi_{2}}\right)\right]+\frac{e^{-\Delta}}{d} \int_{-d / 2}^{d / 2} \cosh \psi(z) d z .
$$

This equation connects the chemical potential $\mu$ with the surface potentials $\psi_{1}$ and $\psi_{2}$. It is the first fundamental equation of the model. In the case in which we consider only adsorption of positive ions, we have to put $A_{+}=A$, and $A_{-} \rightarrow \infty$. In this limiting case Eq. (19) is reduced to Eq. (6) of Ref. [18](for $A_{1}=A_{2}$ ).

The second fundamental equation of the model is obtained in the framework of the Poisson-Boltzmann theory, by means of the Poisson's equation

$$
\frac{d^{2} V}{d z^{2}}=-\frac{q}{\epsilon}\left[n_{+}(z)-n_{-}(z)\right]
$$

because we consider only the steady-state distribution of charges and fields when the applied voltage is held constant. Equation (20) can be put in the form

$$
\frac{d^{2} \psi}{d z^{2}}=\frac{1}{L^{2}} e^{\mu-\Delta} \sinh \psi
$$

where

$$
L=\sqrt{\frac{\epsilon k_{B} T}{2 n_{0} q^{2}}}
$$

is an intrinsic length of the problem. This length is connected to the Debye screening length $\lambda_{D}$ [23] through 
the relation $\lambda_{D}=L e^{\Delta / 2}[10]$. Equation (21) can be integrated to give

$$
\frac{1}{2}\left(\frac{d \psi}{d z}\right)^{2}=\frac{e^{\mu-\Delta}}{L^{2}}[\cosh \psi(z)+c],
$$

where $c$ is an integration constant to be determined by the boundary conditions.

Since the electric field is given by

$$
E(z)=-\frac{d V}{d z}=-\frac{k_{B} T}{q} \frac{d \psi}{d z},
$$

in the presence of an external field the boundary conditions are

$$
\begin{aligned}
E(-d / 2) & =-\frac{k_{B} T}{q}\left(\frac{d \psi}{d z}\right)_{z=-d / 2}=\frac{q}{\epsilon}\left(\sigma_{1}-\Sigma\right), \\
E(d / 2) & =-\frac{k_{B} T}{q}\left(\frac{d \psi}{d z}\right)_{z=d / 2}=-\frac{q}{\epsilon}\left(\sigma_{2}+\Sigma\right),
\end{aligned}
$$

where $\Sigma$ is the surface density of external charges. Equations (25) are written by assuming that the surface at $z=-d / 2$ is connected with the negative pole of the external power supply. The set of equations (19), (21) and (25) furnishes the complete formal solution of the electrostatic problem, giving $\mu, \psi_{1}, \psi_{2}$ and $c$.

In the absence of external field, equations (25) are reduced, respectively, to

$$
E(-d / 2)=q \frac{\sigma_{1}}{\epsilon} \quad \text { and } \quad E(d / 2)=-q \frac{\sigma_{2}}{\epsilon} .
$$

Equations (25) permits to consider two separated cases for which $\sigma_{1}-\Sigma>0$ (low voltage regime) and $\sigma_{1}-\Sigma<0$ (high voltage regime).

When $\sigma_{1}-\Sigma \geq 0, E(-d / 2)>0$ (i.e., $\left.(d \psi / d z)_{z=-d / 2}<0\right)$ and $E(d / 2)<0$ (i.e., $\left.(d \psi / d z)_{z=d / 2}>0\right)$. This implies that the electrical potential has a minimum at some point $z^{*}$ inside the slab, where the electric field vanishes [18], namely

$$
\left(\frac{d \psi}{d z}\right)_{z=z^{*}}=0,
$$

and the integration constant in (23) can be written as

$$
c=-\cos \psi^{*},
$$

where $\psi^{*}=\psi\left(z^{*}\right)$. In this case Eq. (23) can be rewritten as

$$
\frac{d \psi}{d z}=\mp \frac{\sqrt{2}}{L} e^{(\mu-\Delta) / 2} \sqrt{\cosh \psi-\cosh \psi^{*}}
$$

where the sign - refers to the region $-d / 2 \leq z \leq z^{*}$, and + to the region $z^{*} \leq z \leq d / 2$. Equations (29) can be integrated to give
$I\left[\psi^{*}, \psi_{2} ;-\cosh \psi^{*}\right]-I\left[\psi_{1}, \psi^{*} ;-\cosh \psi^{*}\right]=\sqrt{2} \frac{d}{L} e^{(\mu-\Delta) / 2}$

where

$$
I[a, b ; c]=\int_{a}^{b} \frac{d \psi}{\sqrt{\cosh \psi+c}} .
$$

In this manner the boundary conditions (25) can be rewritten as

$$
\begin{aligned}
& \frac{\sqrt{2} k_{B} T}{q^{2} L} e^{(\mu-\Delta) / 2} \sqrt{\cosh \psi_{1}-\cosh \psi^{*}}=\frac{\sigma_{1}-\Sigma}{\epsilon}, \\
& \frac{\sqrt{2} k_{B} T}{q^{2} L} e^{(\mu-\Delta) / 2} \sqrt{\cosh \psi_{2}-\cosh \psi^{*}}=\frac{\sigma_{2}+\Sigma}{\epsilon} .
\end{aligned}
$$

The fundamental equations of the model for the low voltage regime are then (19), (30) and (32). We have to solve this system of four equations to obtain $\mu, \psi_{1}$, $\psi_{2}$ and $\psi^{*}$. Once this system of equations is solved, it is straightforward to obtain the surface charge densities $\sigma_{i}$ by means of Eqs. (13). As it follows from these equations, the surface charge densities depend on the external charges at the surface through the chemical potential and the electric potentials at the surfaces.

The border separating the two regimes is defined by $\sigma_{1}\left(\Sigma_{c}\right)-\Sigma_{c}=0$, where $\Sigma_{c}$ is the critical surface density of external charges. For $\Sigma=\Sigma_{c}, \psi^{*}\left(\Sigma_{c}\right)=\psi_{1}\left(\Sigma_{c}\right)$, as it follows from Eqs. (32). In the high-voltage regime the adsorbed charge, at $z=-d / 2$, is then smaller than the one sent by the power supply on the electrode. From Eqs. (25) we now have that $E(-d / 2)<0$ and $E(d / 2)<0$. The electrical potential is a monotonic function of $z$ and, consequently, the electric field never vanishes for $-d / 2 \leq z \leq d / 2$ [18]. In this case, from Eq. (23) we obtain

$$
I\left[\psi_{1}, \psi_{2} ; c\right]=\sqrt{2} \frac{d}{L} e^{(\mu-\Delta) / 2},
$$

connecting $c$ to $\psi_{1}$ and $\psi_{2}$. By using Eqs. (23) and (25) we deduce that the boundary conditions read

$$
\begin{aligned}
& \frac{k_{B} T}{q} \frac{\sqrt{2}}{L} \sqrt{\cosh \psi_{1}+c}=q \frac{\Sigma-\sigma_{1}}{\epsilon} \\
& \frac{k_{B} T}{q} \frac{\sqrt{2}}{L} \sqrt{\cosh \psi_{2}+c}=q \frac{\Sigma+\sigma_{2}}{\epsilon} .
\end{aligned}
$$

In the high-voltage regime, the fundamental equations are (19), (33) and (34). These equations give $\mu, \psi_{1}, \psi_{2}$ and $c$ in terms of $\Sigma$ and $d$. 

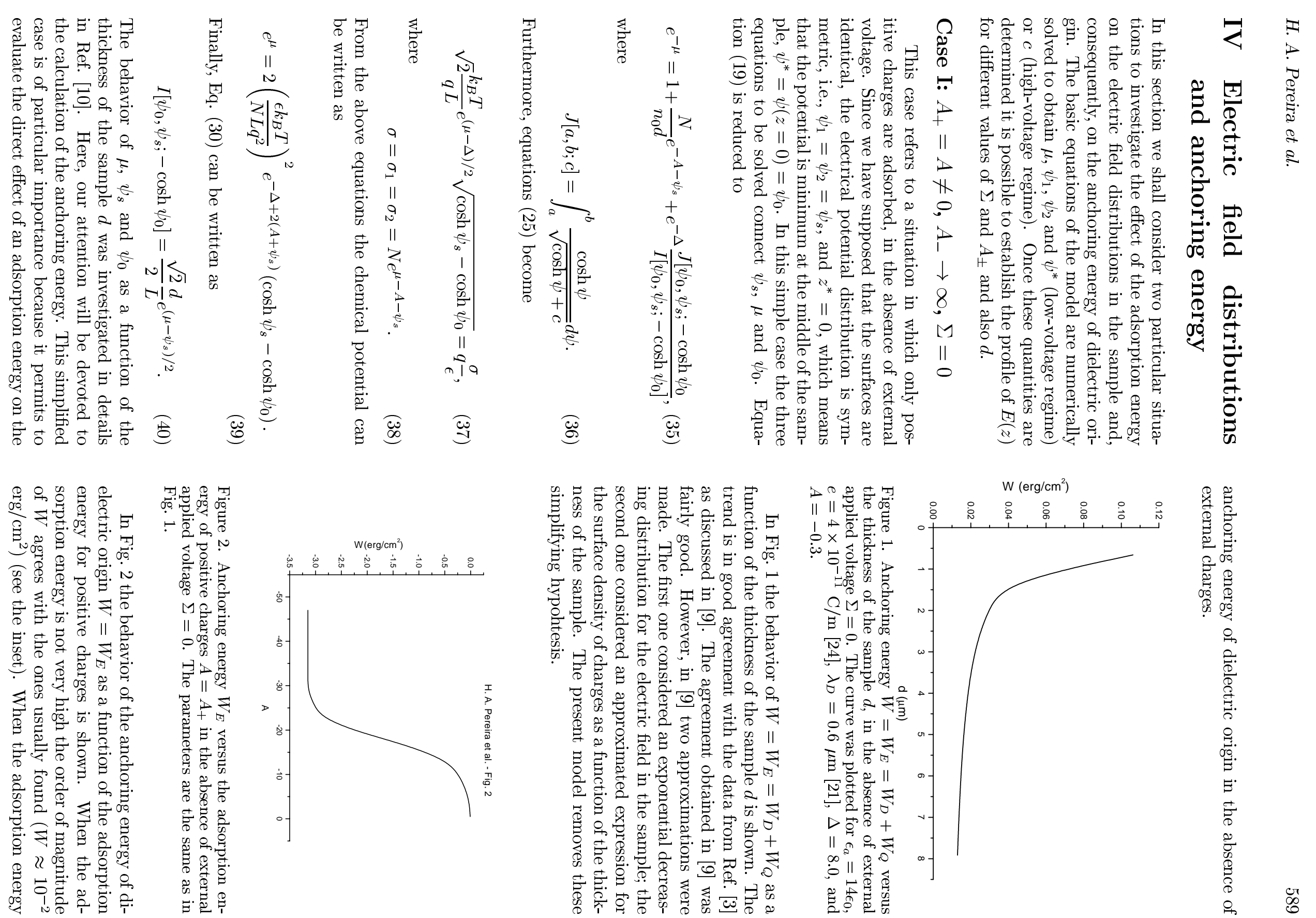
is very high, $W$ tends to a constant value, independent of the value of $A$. This value is of the order of few $\mathrm{erg} / \mathrm{cm}^{2}$ and corresponds practically to a strong anchoring situation. This result indicates again that the ionic adsorption can play a fundamental role in establishing the correct order of magnitude of $W$.

\section{Case II: $A_{+}=A \neq 0, A_{-} \rightarrow \infty, \Sigma \neq 0$}

In this case, we have again only adsorption of positive charges, but now in the presence of an external voltage. In Fig. 3 we present an illustrative result when the external density of charge is $\Sigma / N=0.6$. The figure shows the behavior of $W=W_{E}$ as a function of $d$. This situation has to be compared with the one depicted in Fig. 1 where the external charges are absent. Notice that the effect of an external electric field strongly affects the magnitude of the anchoring energy. This result is in complete agreement with the ones established in [19], where it was demonstrated that the anchoring energy is bias-voltage dependent.

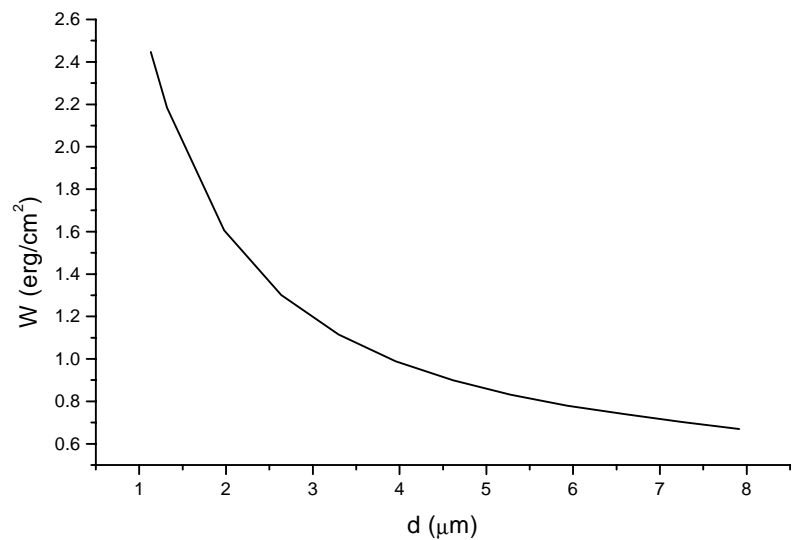

Figure 3. The same as in Fig. 1, in the presence of external applied voltage $\Sigma / N=0.6$. The parameters are the same as in Fig. 1.

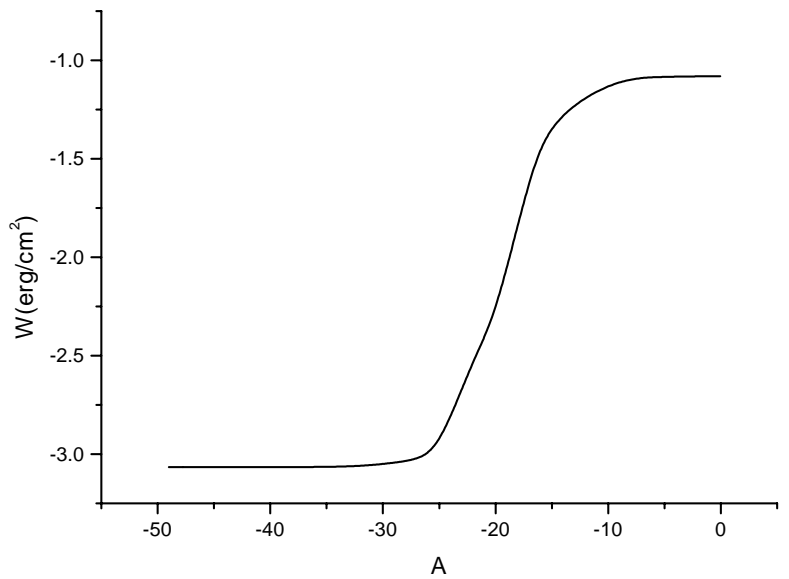

Figure 4. The same as in Fig. 2, in the presence of external applied voltage $\Sigma / N=0.6$. The parameters are the same as in Fig. 1.
In Fig. 4 the quantity $W=W_{E}$ is plotted as a function of the adsorption energy $A$, also in the presence of an external charge density $\Sigma / N=0.6$. This figure illustrates the combined effect of an external voltage and an adsorption energy in the behavior of $W=W_{E}$. Both effects act to increase the magnitude of $W$. Again we have a saturation value for $W$ for large values of $A$, corresponding to a situation of strong anchoring (when $A \rightarrow-\infty)$.

In Fig. 5 the anchoring energy $W=W_{E}$ is shown as a function of the difference of potential across the sample. $\Delta \psi=\psi_{2}-\psi_{1}$ is the effective difference of potential, i.e., it comes from the external charges and the internal charges that move to the surface. The value of $W$ is an increasing function of this difference of potential, but presents a maximum near $\Delta \psi=50$, i.e., $\Delta V \simeq 1.25 \mathrm{~V}$ (for monovalent ions). For large difference of potential the anchoring energy of dielectric origin tends to a saturation value. The curve was plotted for an adsorption energy $A=-0.4$.

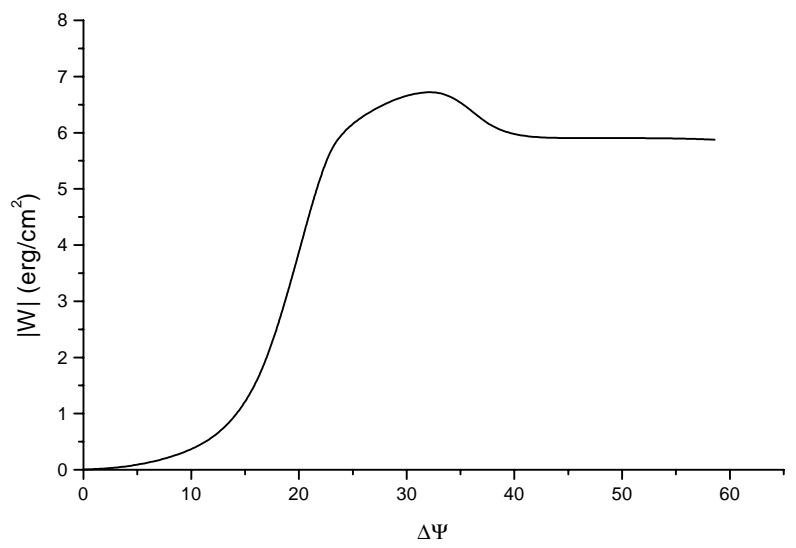

Figure 5. Anchoring energy of dielectric origin $W_{E}$ as a function of the effective potential difference across the sample $\Delta \psi=\psi_{2}-\psi_{1}$, for $A_{+}=-0.3$ and $A_{-} \rightarrow \infty$. The parameters are the same as in Fig. 1.

Finally, just to show the effect of the adsorption of negative charges on the net surface charge density, this quantity is exhibited as a function of the thickness of the sample in two cases - in the absence of external applied voltage. In Fig. $6 \sigma / N=\sigma_{1} / N=\sigma_{2} / N$ is shown as function of the $d$ for the case $A_{+}=-0.4$ and $A_{-} \rightarrow \infty$. In this case, only the adsorption of positive charges is considered. One observes that the behavior of $\sigma$ is linear with $d$, for small $d$, and tends to a value which is independent of $d$, for very large values of $d$, as discussed in [10]. In Fig. 7 the same quantity is exhibited as a function of $d$ for the case $A_{+}=-0.4$ and $A_{-}=-1.0$ The global behavior is similar, in the sense that there is a linear behavior for small $d$ and a saturation value for large $d$. However, as expected, the order of magnitude of the density in this case is less than in the preceding figure, because the net charge density is 
given by $\sigma=\sigma_{+}-\sigma_{-}$(see Eq. (15)). Furthermore, in this situation $\sigma$ tends to a saturation value only for very large values of $d$, as compared with the previous case where the saturation is abrupt.

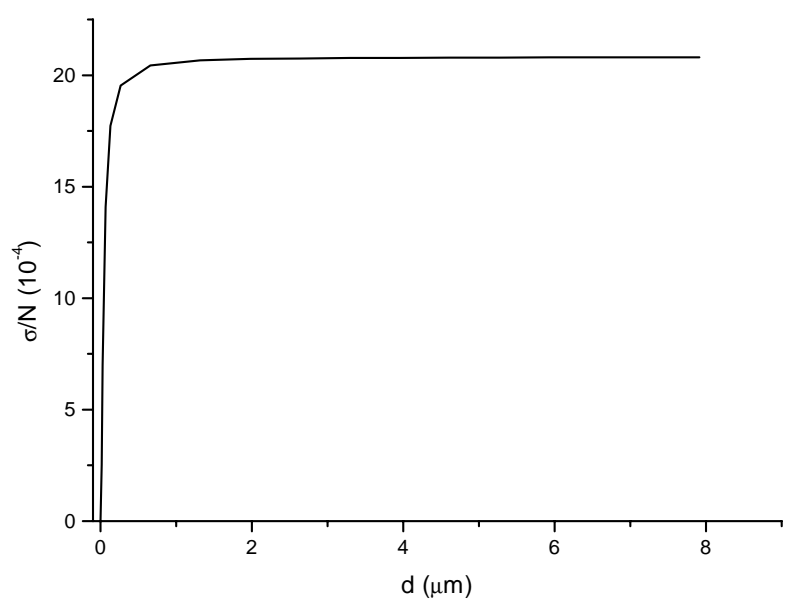

Figure 6. Surface charge density $\sigma$ versus the thickness of the sample in the case of adsorption of only positive charges $A_{+}=-0.4\left(A_{-} \rightarrow \infty\right)$. The parameters are the same as in Fig. 1.

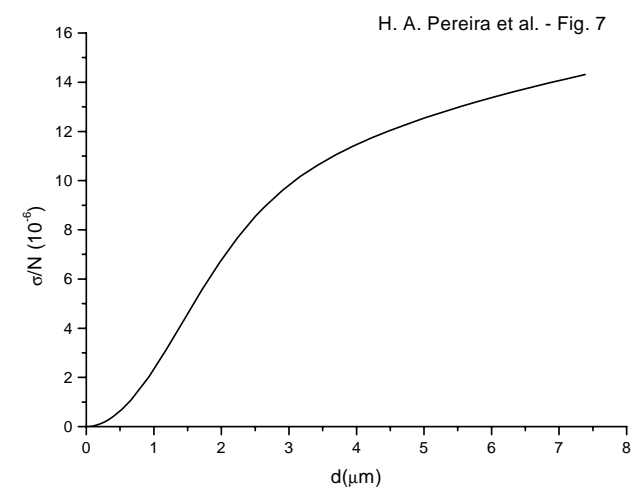

Figure 7. Surface charge density $\sigma$ versus the thickness of the sample in the case of adsorption of positive (with $A_{+}=-0.4$ ) and negative (with $A_{-}=-1.0$ ) charges. The parameters are the same as in Fig. 1.

A more extensive analysis consists in taking into account also the effect of the adsorption of negative charges on the anchoring energy. Due to the generality of the proposed model, it is also possible to consider different surfaces, with different adsorption energies for each species of ions. This will permit a complete overview on the main predictions of the model. This analysis is under progress and will be published elsewhere.

\section{Conclusions}

We have analyzed the effect of the selective ionic adsorption on the anchoring energy of a nematic liquid crystal sample. The problem of a sample of thickness $d$ formed by two identical surfaces, under the action of an external applied voltage was analyzed. It was shown that the adsorption energy has a profound effect on the magnitude of the anchoring energy. Our analysis reinforces the conclusion that the anchoring energy depends on the external applied voltage, in agreement with the predictions of Ref. [19]. Furthermore, we have shown that, also in the absence of external field, the anchoring energy is strongly influenced by the adsorption energy of the ions. The anchoring energy presents a nonmonotonic behavior as a function of the adsorption energy. For very large values of this quantity, the anchoring energy tends to a saturation value whose magnitude is of the order of a few erg $/ \mathrm{cm}^{2}$. In the cases we have analyzed, the presence of the flexoelectricity was explicitly taken into account, and plays a dominant role. In conclusion, to investigate the correct behavior of the anchoring energy in a real sample one has to consider the renormalization of the anchoring energy of dielectric origin. This contribution results from the coupling of spatial dependent electric field inside the sample originated from the ionic adsorption- with the dielectric and flexoelectric properties of the medium.

\section{Acknowledgments}

This work has been partially supported by Fundação Araucária, Capes, and INFM.

\section{References}

[1] P. J. Coolings, Liquid Crystals, (Adam Hilger, Bristol, 1990), Chapters 6 and 7.

[2] G. Barbero and L. R. Evangelista, An Elementary Course on the Continuum Theory for Nematic Liquid Crystals, (World Scientific, Singapore, 2000).

[3] L. M. Blinov, A. Yu. Kabaenkov, and A. A. Sonin, Liq. Cryst. 5, 645 (1989).

[4] O. A. Gomes, R. C. Falcão, and O. N. Mesquita, Phys. Rev. Lett. 86, 2577 (2001)

[5] G. Strangi, C. Versace, and N. Scaramuzza, Appl. Phys. Lett. 78, 2455 (2001).

[6] B. Jerome, Rep. Prog. Phys. 54, 391 (1991).

[7] G. Barbero and G. Durand, Liq. Cryst. 2, 401 (1982).

[8] G. Barbero and G. Durand, J. Phys. (France) 51, 281 (1990).

[9] A. L. Alexe-Ionescu, G. Barbero, and A. G. Petrov, Phys. Rev. E 48, R1631 (1993).

[10] G. Barbero, A. K. Zvezdin, and L. R. Evangelista, Phys. Rev. E 59, 1846 (1999).

[11] V. G. Nazarenko and O. D. Lavrentovich, Phys. Rev. E 49, R990 (1994). 
[12] U. Kühnau, A. G. Petrov, G. Klose, and H. Schmiedel, Phys. Rev. E 59, 578 (1999).

[13] V. G. Nazarenko, V. M. Pergamenshchik, O. V. Koval'chuk, A. B. Nych, and B. I. Lev, Phys. Rev. E 60, 5580 (1999).

[14] V. U. Fazio, and L. Komitov, Europhys. Lett. 46(1), 38 (1999).

[15] R. Meister, and B. Jérôme, J. Appl. Phys. 86, 2473 (1999).

[16] A. L. Alexe-Ionescu, A. T. Ionescu, N. Scaramuzza, G. Strangi, C. Versace, G. Barbero, and R. Bartolino, Phys. Rev. E 011708-1 (2001).

[17] K. Bohinc, V. Kralj-Iglic, and A. Iglic, Electrochimica Acta 46, 3033 (2001).
[18] L. R. Evangelista and G. Barbero, Phys. Rev. E 64, 021101-1, (2001).

[19] D. Olivero, L. R. Evangelista, and G. Barbero, Phys. Rev. E 65, 031721 (2002).

[20] R. N. Thurston, J. Cheng, R. B. Meyer, and G. D. Boyd, J. Appl. Phys. 56, 264 (1984).

[21] R. N. Thurston, J. Appl. Phys. 55, 4154 (1984).

[22] L. D. Landau and E. I. Lifshitz, Electrodynamique des Milieux Continus, (MIR, Moscow, 1956).

[23] J. Israelachvili, Intermolecular Forces (Academic Press, London, 1985), Chap. 12.

[24] G. Barbero, A. N. Chuvyrov, A. P. Krekhov, and O. A. Scaldin, J. Appl. Phys. 69, 6343 (1991). 